\title{
ANÁLISIS DESCRIPTIVO DE LA INFLUENCIA DE LOS FACTORES SOMATOMÉTRICOS EN EL NEURODESARROLLO DE LOS MENORES CON CARDIOPATÍAS CONGÉNITAS
}

\author{
Descriptive Analysis of the Influence of Somatometric Factors in the Neurodevelopment \\ of Minors with Congenital Heart Diseases
}

\author{
Maria del Rosario Mendoza Carretero ${ }^{1}$, Susana Ares Segura ${ }^{2}$, \\ Leticia Albert de la Torre ${ }^{3}$, Marta Medina del Río ${ }^{4}$, Belén Sáenz-Rico ${ }^{1}$ \\ ${ }^{1}$ Universidad Complutense de Madrid, España \\ ${ }^{2}$ Hospital Universitario La Paz de Madrid, España \\ ${ }^{3}$ Hospital 12 de Octubre, España \\ ${ }^{4}$ Fundación Menudos Corazones, España
}

\section{KEY WORDS}

Congenital Heart Disease Neurodevelopment Biomedical Criteria Early Childhood
ABSTRACT

Somatometric measurements of the newborn are related to their gestational age. We want to know if the biomedical criteria are related to the slowdowns found in the neurodevelopment of minors with congenital heart defects (CC). There were 49 participants, who were assessed through the Bayley-III Child Development Scale. The results indicate that the somatometric criteria, which acquire an incidence in premature infants, seem not to be involved in the slowdowns of the population with CHD. It is necessary to expand the study sample and include incident biomedical variables in the CC to know the degree of relationship.

\section{PALABRAS CLAVE}

Cardiopatías congénitas

Neurodesarrollo

Criterios biomédicos

Primera infancia

\section{RESUMEN}

Las medidas somatométricas del recién nacido se relacionan con su edad gestacional. Se quiere conocer si los criterios biomédicos están relacionados con las lentificaciones encontradas en el neurodesarrollo de los menores con cardiopatías congénitas (CC). Se contó con 49 participantes, que fueron valorados a través de la Escala de Desarrollo Infantil Bayley-III. Los resultados indican que los criterios somatométricos, que adquieren incidencia en los prematuros, parecen no estar implicados en las lentificaciones de la población con CC. Es necesario ampliar la muestra del estudio e incluir variables biomédicas incidentes en las CC para conocer el grado de relación.

Recibido: 06/08/2019

Aceptado: 21/04/2020

\section{GLOBAL KNOWLEDE}




\section{Introducción}

$\mathrm{L}$ as cardiopatías congénitas (CC) son malformaciones del corazón o de los grandes vasos sanguíneos, presentes en el feto y en el recién nacido (MMCC, 2019). La mayoría de estas anomalías se pueden diagnosticar intraútero a través de la ecocardiografía fetal y tras sospecha previa en las ecografías convencionales. A pesar de esto, algunas cardiopatías congénitas como la coartación de la aorta o las comunicaciones interventriculares, son difíciles de descartar debido a la circulación fetal. El origen de estas anomalías requiere especial atención pues la Sociedad Española de Cardiología Pediátrica y Cardiopatías Congénitas (2014) señala que pueden ser hereditarias (5-8\%), de estas el 5\% están vinculadas a anomalías cromosómicas como el Síndrome de Down (trisomía 21), Síndrome de Edwars ${ }^{1}$ (trisomía 18), el Síndrome DiGeorge $^{2}$, ligado al cromosoma 22q11, entre otros; y el 3\% a defectos del gen único. El 2\% se corresponderían a factores ambientales y a enfermedades maternas aunque en la mayoría de los casos (85-90\%) se desconoce su aparición pero el riesgo puede verse incrementado cuando uno de los progenitores presenta cardiopatía congénita.

Las anomalías cardiacas se clasifican según su anatomía y según su fisiopatología (Albert, 2019). La primera es la más utilizada por los especialistas, pues se describe la morfología de las aurículas, ventrículos, válvulas aurículoventriculares y posición de los vasos. La segunda manifiesta su característica clínica más significativa, dividiéndose en cianóticas, si generan niveles bajos de oxígeno en la sangre, lo cual provoca un color azulado en la piel y en la membrana mucosa (MedlinePlus, 2019) y acianóticas, las cuales aparecen sin disminución de la saturación de oxígeno en la sangre, encontrándose las CC con cortocircuito de

\footnotetext{
${ }^{1}$ El Síndrome de Edwars es una enfermedad cromosómica rara caracterizada por la presencia de un cromosoma adicional en el par 18. Su frecuencia es de 1 de cada 6000 a 1 de cada 13000 nacidos vivos. Aparece con mayor frecuencia en las mujeres (AAMADE, 2012).

2 El Síndrome de DiGeorge es un trastorno causado por una deleción en el cromosoma 22, lo que genera un desarrollo insuficiente en varios sistemas del cuerpo (Mayo Clinic, 2019).
}

izquierda a derecha, las obstructivas y las miocardiopatías (Fundación Salud Infantil, 2019).

A continuación, se presenta la clasificación de los tipos de cardiopatías congénitas en función de su fisiopatología.

Tabla 1. Tipos de cardiopatías congénitas en función de su fisiopatología

\begin{tabular}{|c|c|c|}
\hline \multirow[t]{3}{*}{$\begin{array}{l}\mathrm{CC} \\
\text { Cianóticas }\end{array}$} & $\begin{array}{l}\text { Obstructivas } \\
\text { corazón } \\
\text { derecho }\end{array}$ & $\begin{array}{l}\text { Tetralogía de Fallot, } \\
\text { atresia pulmonar, } \\
\text { ventrículo único o } \\
\text { atresia tricuspídea } \\
\text { con estenosis } \\
\text { pulmonar. }\end{array}$ \\
\hline & Mezcla total & $\begin{array}{l}\text { Ventrículo único o } \\
\text { atresia tricuspídea } \\
\text { sin estenosis } \\
\text { pulmonar, truncus } \\
\text { arterioso, drenaje } \\
\text { venoso anómalo } \\
\text { pulmonar total. }\end{array}$ \\
\hline & $\begin{array}{ll}\text { Falta } & \text { de } \\
\text { Mezcla } & \\
\end{array}$ & $\begin{array}{l}\text { Trasposición de } \\
\text { Grandes Arterias }\end{array}$ \\
\hline \multirow[t]{3}{*}{$\begin{array}{l}\text { CC } \\
\text { Acianóticas }\end{array}$} & $\begin{array}{l}\text { Cortocircuito } \\
\text { de izquierda a } \\
\text { derecha }\end{array}$ & $\begin{array}{l}\text { Comunicación } \\
\text { interventricular, } \\
\text { comunicación } \\
\text { interauricular, } \\
\text { ductus, canal } \\
\text { aurículo- } \\
\text { ventricular, drenaje } \\
\text { venoso anómalo } \\
\text { pulmonar parcial. }\end{array}$ \\
\hline & $\begin{array}{l}\text { Obstructivas } \\
\text { corazón } \\
\text { izquierdo }\end{array}$ & $\begin{array}{lr}\text { Coartación } & \text { aórtica, } \\
\text { estenosis } & \text { aórtica, } \\
\text { estenosis } & \text { mitral, } \\
\text { hipoplasia } & \\
\text { ventrículo } & \\
\text { izquierdo. } & \\
\end{array}$ \\
\hline & $\begin{array}{l}\text { Insuficiencias } \\
\text { valvulares y } \\
\text { otras }\end{array}$ & $\begin{array}{l}\text { Insuficiencia mitral, } \\
\text { insuficiencia } \\
\text { aórtica, estenosis } \\
\text { ramas pulmonares. }\end{array}$ \\
\hline
\end{tabular}

Fuente: Fundación Salud Madrid (2019).

Las anomalías cardiacas presentan una tasa de incidencia de 8 de cada 1000 nacidos vivos en España (Salamanca-Zarzuela et al., 2018), y son la causa principal de una elevada mortalidad neonatal en los países en desarrollo (PérezLescure et al., 2017). Sin embargo, al menos, el $85 \%$ de los menores con CC llegan a la edad adulta (Brotons, 2014) y gozan de una buena 
calidad de vida (SECPCC, 2014). Esto se debe a los avances quirúrgicos experimentados por la cirugía cardiaca desde los años 40 y a los cuidados perioperatorios surgidos en las últimas décadas (Wiliams et al., 2015); ya que en la actualidad todas las CC, gozan de intervención paliativa y/o correctora.

Tras garantizar la supervivencia de los menores con CC, la literatura al respecto, apunta que se empiezan a evidenciar morbilidades en el neurodesarrollo en estos menores (MartínezBiarge, 2013), que pueden estar relacionadas con factores biomédicos como la anestesia, la cirugía por circulación extracorpórea ${ }^{3}$ (CEC), el peso, la edad gestacional, la longitud y el perímetro cefálico, presentando una etiología multifactorial (Martínez-Biarge, 2013).

A esto se le debe añadir que los menores cardiópatas pueden presentar un crecimiento inferior al resto de la población (Solar y García, 2010), lo cual puede estar relacionado con un signo de insuficiencia cardiaca ya sea por carecer de intervención quirúrgica o bien, porque presenten lesiones residuales. Su desarrollo psicomotor puede verse alterado, especialmente, si han sido sometidos a una cirugía prolongada por circulación extracorpórea y presentan riesgo de sufrir endocarditis ${ }^{4}$. A este respecto se aconseja que las hospitalizaciones no sean prolongadas para que puedan integrarse completamente en las actividades escolares.

Recurriendo a los resultados contrastados en la literatura científica (Salamanca-Zarzuela et al., 2018), se estima la necesidad de conocer si los criterios biomédicos están o no relacionados con las lentificaciones encontradas en las áreas del desarrollo comunicativo-lingüística y motora de los menores con CC, sin anomalías cromosómicas asociadas.

Antes de proceder a mostrar la metodología utilizada, los resultados alcanzados $\mathrm{y}$ las conclusiones obtenidas, se considera necesario arrojar información sobre las cardiopatías congénitas más frecuentes en el estudio y las

\footnotetext{
${ }^{3}$ La cirugía por circulación extracorpórea, también conocida como operación abierta, permite detener el corazón del menor durante la intervención mientras que máquina funciona como corazón y pulmón artificial (SECPCC, 2014).

${ }^{4}$ Endocarditis: infecciones del interior del corazón (SECPCC, 2014).
}

(co)morbilidades que pueden generarse en el ámbito escolar.

\section{Cardiopatías congénitas más frecuentes en el estudio}

Las cardiopatías congénitas se pueden clasificar según su anatomía y según su fisiopatología. En este caso, se tiene por consideración la última categorización, la cual las divide en cianóticas y acianóticas. Las primeras se corresponden con la Tetralogía de Fallot (TOF), Coartación de la Aorta (CoA) y la Transposición de las grandes arterias o de los grandes vasos (TGA), etc.; las segundas hacen referencia a la Comunicación Interventricular (CIV), Comunicación Interauricular (CIA), Canal Auriculoventricular Común (CAV), entre otras.

Si bien es cierto, la mayoría de la muestra de la población representada en este estudio cuentan con TGA, CIV y CIA. Con base a esto, se considera necesario a la par que preciso, aunque no se corresponda con el objetivo de este estudio, explicar brevemente cada una de las cardiopatías más frecuentes mencionadas pero para ello es conveniente conocer brevemente el funcionamiento del corazón normal.

El corazón sano es la unión de dos circulaciones, la pulmonar (derecha) que es la encargada de enviar la sangre sin oxígeno al pulmón para que se oxigene y la sistémica (izquierda) que envía la sangre oxigenada a todo el cuerpo. Cada circulación está formada por dos cavidades conocidas como aurículas derecha e izquierda, las cuales se encargan de recoger la sangre del cuerpo o del pulmón a través de las venas, y por los ventrículos, izquierdo y derecho; el primero impulsa la sangre a todo el cuerpo y el último, la impulsa hacia el pulmón (Área del Corazón Infantil HGUGM, 2017).

La Transposición de las Grandes Arterias es una malformación en la que existe una alteración en la conexión del ventrículo arterial (Quero, M. y Sánchez, I., 2006) ya que la aorta y la arteria pulmonar están intercambiadas (MedlinePlus, 2019), generando dificultad respiratoria y provocando cianosis.

La Comunicación Interventricular puede localizarse en el tabique interventricular, en el cual hay un orificio que separa los dos 
ventrículos. Esto hace que la sangre oxigenada pase al ventrículo izquierdo, a través de ese agujero, y después se mezcle en el ventrículo derecho con la sangre pobre en oxígeno (Children's Health, 2019) y la Comunicación Interauricular conlleva una abertura en la pared que divide las dos cavidades superiores del corazón (aurículas derecha e izquierda). La sangre oxigenada pasa desde la aurícula izquierda, a través del hueco del tabique, y se mezcla con la sangre pobre en oxigeno en la aurícula derecha (Children's Health, 2019).

\section{Morbilidades y comorbilidades ámbito educativo}

Según Dittrich et al. (2003) y Matsuzaki et al. (2010), los menores con CC presentan lentificaciones en el desarrollo motor y en el área comunicativa-lingüística que pueden derivar en morbilidades y comorbilidades en edades posteriores, las cuales se hacen más palpables en la etapa escolar. Estas pueden estar causadas por la duración de la intervención quirúrgica mediante cirugía por circulación extracorpórea (CEC), la prolongación de la estancia hospitalaria, entre otras (Marino et al., 2012).

Sin embargo, antes de vislumbrar las comorbilidades en el ámbito escolar, se considera necesario mencionar distintos estudios como el de Chen et al. (2015) que muestran que los menores con CC $(\mathrm{N}=10)$ obtuvieron puntuaciones inferiores, aunque no significativas, en el dominio motor en comparación con el grupo de control sano $(\mathrm{N}=14)$ cuando fueron evaluados a los tres meses de edad a través de la Escala de Desarrollo Infantil Bayley-III $(95,86 \pm 10,26-90.10 \pm 7.36$, respectivamente). Dentro del área motora, estudios como el de Sananes et al. (2012) $(\mathrm{N}=106)$ evidencian que la motricidad fina tiene un desarrollo inferior cuánta mayor edad tiene el niño con CC mientras que la motricidad gruesa tiende a normalizarse con el desarrollo evolutivo del menor. Para mostrar estos resultados valoraron, a través de la escala mencionada con anterioridad, a infantes con cardiopatías congénitas de 8,12 y 24 meses de edad aunque las pruebas que se realizan en la primera infancia tienen una validez limitada (McGrath, 2004) y sería necesario realizarlas en distintos lapsos temporales (24 meses, de los 36 a 60 meses y de los 132 a los 144 meses) según la Asociación Americana del Corazón.

Regresando a las dificultades halladas en el periodo escolar, autores como Schaefer et al. (2013) y Miatton et al. (2007) evidencian que los niños diagnosticados de Tetralogía de Fallot (TOF) obtienen menores puntuaciones en la lectura, ortografía y aritmética, necesitando apoyos en estas áreas. Aquellos menores con cardiopatía isquémica compleja obtienen puntuaciones inferiores a la media en el funcionamiento ejecutivo, en el desempeño escolar y en la regulación de la conducta (Gerstle et al., 2016). Junto a esto, Rollins y Newburguer (2013) indican que los padres de los niños con CC reportaron retrasos en el desarrollo de las frases, en los gestos y en la comprensión de vocabulario.

Se puede creer que los menores presentan estos resultados debido a la falta de asistencia a la escuela por motivos médicos pero no es así pues muchos de los niños, una vez iniciada la escolarización, acuden a la escuela con regularidad (Schaefer et al., 2013).

Aunque, se puede pensar que, la regulación conductual puede no estar vinculada a las lentificaciones encontradas en las área del desarrollo, se considera fundamental y necesario tenerlo en consideración pues está comprobado que la regulación de las emociones pueden afectar e influir en el desarrollo escolar de estos niños (Sarrechia et al., 2015; Goldsworthy et al., 2016). Y al parecer, según investigaciones previamente realizadas, estos infantes obtienen un desarrollo inferior en la regulación conductual, lo cual produce problemas de internalización y externalización aunque el grado varía en función del tipo de cardiopatía pues los que presentaban Síndrome Izquierdo Hipoplásico alcanzaban peores resultados que los que habían sido diagnosticados de Transposición de las Grandes Arterias (TGA) (Gerstle et al., 2016).

A pesar de esto, los resultados obtenidos no pueden generalizarse a toda la población pues es necesario continuar investigando sobre los rasgos conductuales de esta población para clarificar y poder generalizar los resultados. 


\section{Metodología}

Esta investigación forma parte del estudio de tesis doctoral que se está realizando en la Facultad de Educación perteneciente a la Universidad Complutense de Madrid (UCM), cuyo título es "Detección, despistaje e intervención de alteraciones del neurodesarrollo en menores con cardiopatías congénitas al nacimiento".

Este estudio, cualitativo, transversal $\mathrm{y}$ multicéntrico, cuenta con una muestra total de $\mathrm{N}=48$, la cual presenta cardiopatías congénitas, sin anomalías cromosómicas y/o síndromes asociados, con edades inferiores a los 42 meses de edad, han sido intervenidos por cirugía por circulación extracorpórea antes de los 12 meses y valorados a través de la Escala de Desarrollo Infantil Bayley-III, con la finalidad de identificar morbilidades y comorbiliadades en el área cognitiva, comunicativa-lingüística y motora.

La cohorte se recogió en un lapso temporal de dos años, desde el 2016 hasta el 2018, en el Hospital Universitario La Paz y el Hospital 12 de Octubre, ambos pertenecientes a la Comunidad de Madrid y referentes en la atención a la Cardiología Pediátrica. Junto a ellos, se suma la Fundación Menudos Corazones (MMCC),ubicada en Madrid, la cual acompaña y ayuda a las familias desde el momento que reciben el diagnóstico de la patología, tanto en su vida cotidiana como durante las estancias hospitalarias (MMCC, 2019).

El procedimiento que se siguió para la recogida de muestra fue el siguiente; el estudio se sometió a la aprobación de la comisión bioética del Comité Científico de Investigación, así como por parte del patronato en la Fundación Menudos Corazones, y, a su vez, con el consentimiento informado de los progenitores. Durante las valoraciones se recopilaron los datos biomédicos al nacimiento, a través del primer informe de neonatología, y los de desarrollo.

Teniendo presente que, según la literatura científica, los patrones de desarrollo de los infantes prematuros son similares a los de los menores con cardiopatías congénitas, se seleccionaron variables biomédicas propias de los primeros (peso, talla, perímetro cefálico, edad gestacional) (Esquivel-Hernández et al., 2013).
Tras los resultados encontrados en las mismas, se incorporaron nuevos criterios biomédicos, como la duración de la cirugía por circulación extracorpórea, el clampaje, el tiempo de ingreso en UCIN y el lapso temporal transcurrido desde el momento de la intervención hasta el momento de la valoración, cuyos datos fueron analizados en una submuestra de 12 participantes, perteneciente a la cohorte total de 49 participantes.

Todos los datos fueron analizados mediante estadísticos descriptivos a través de Microsoft Excel, a fin de obtener una precisa representación de las puntuaciones alcanzadas tanto en los criterios biomédicos (peso, talla, perímetro cefálico, edad gestacional) como en los de desarrollo (cognitivo, motor y lenguaje).

Para ello, se siguió el siguiente procedimiento. En primer lugar, se analizaron los criterios biomédicos y de desarrollo de la totalidad de la muestra $(\mathrm{N}=48)$. En segundo lugar, se seleccionaron aquellos sujetos cuya edad gestacional (EG) se situaba entre las semanas $37 \mathrm{y}$ 40, pues se consideran nacimientos a término. En tercer, y último lugar, se examinaron las puntuaciones obtenidas en aquellos participantes que nacieron en las semanas 35,36 y 41 ya que se clasifican como nacimientos tempranos y tardíos, respectivamente. Los análisis se realizaron a través de medidas de tendencia central, concretamente usando la media.

\section{Resultados}

La muestra del presente estudio se encuentra dentro de los parámetros de referencia de Kids Heath (2018), pues indica que los bebés nacidos entre las semanas 37 y 40 de gestación pesan entre $2.5 \mathrm{~kg}$ y $4 \mathrm{~kg}$, aproximadamente, y de la OMS (2019) que señala que presentan una talla entre $46 \mathrm{~cm}$ y $53,5 \mathrm{~cm}$ aproximadamente, mientras que los del perímetro cefálico están situados entre 32 y $35 \mathrm{~cm}$.

En el primer análisis, los resultados de la totalidad de la muestra $(\mathrm{N}=48)$ evidencian que los criterios biomédicos se sitúan dentro del promedio de la población (EG $\overline{\mathbf{X}}=\mathbf{3 8}$, peso $\bar{X}=3.079$ g., talla $\bar{X}=\mathbf{4 8} \mathbf{c m}$ y $P C \bar{X}=\mathbf{3 2}, 8 \mathbf{c m}$ ) aunque existen lentificaciones en el desarrollo del lenguaje $(\overline{\mathbf{X}}=\mathbf{8 7}, 6)$ y a nivel motor $(\overline{\mathbf{X}}=\mathbf{8 8}, \mathbf{7})$. 
En el segundo análisis se seleccionaron a aquellos sujetos $(\mathrm{N}=44)$ cuya $\mathrm{EG}$ estaba entre las semanas 37-40, obteniéndose resultados parecidos a los anteriores (EG $\overline{\mathbf{X}}=38,5$; peso $\overline{\mathbf{X}}=\mathbf{3 . 1 3 7}$ g.; talla $\overline{\mathrm{X}}=\mathbf{4 8 , 2} \mathrm{cm}, \quad \mathrm{PC} \overline{\mathrm{X}}=\mathbf{3 3}, \mathbf{8 6} \mathrm{cm}$, lenguaje $\overline{\mathbf{X}}=\mathbf{8 7}, \mathbf{6}$ y motor $\overline{\mathbf{X}}=\mathbf{8 9}$ ) y en el tercer análisis $(\mathrm{N}=4)$ los resultados son similares en el neurodesarrollo de aquellos cuya EG fue de 35,36 y 41 semanas (lenguaje $\overline{\mathbf{X}}=\mathbf{8 7}, \mathbf{6}$ y motor $\overline{\mathbf{X}}=$ $\mathbf{8 5}, 6)$; a diferencia del resto, tanto la talla como el PC se encontraban por debajo del promedio.

Gráfico 1. Resultados de los análisis de los criterios biomédicos en menores con cardiopatías congénitas.

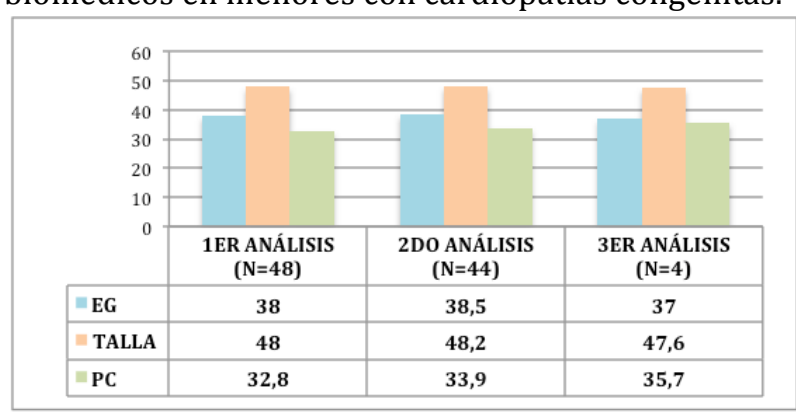

Fuente: elaboración propia.

Los resultados obtenidos entre los criterios biomédicos y su influencia en las áreas de desarrollo, correspondientes a la muestra con cardiopatía congénita, evidencian que no existen diferencias con los alcanzados en la población sin este tipo de alteraciones en el funcionamiento. A pesar de esto, se observan lentificaciones en el neurodesarrollo, siguiendo la clasificación cualitativa de la Escala de Desarrollo InfantilBayley III, concretamente en el área del lenguaje y en el desarrollo motor en aquellos menores con malformaciones en los grandes vasos sanguíneos.

Tabla 2. Parámetros de cociente de desarrollo y comorbilidad según Bayley-III

\begin{tabular}{|l|l|}
\hline $\begin{array}{l}\text { Puntuaciones } \\
\text { compuestas }\end{array}$ & $\begin{array}{l}\text { Clasificación } \\
\text { cualitativa }\end{array}$ \\
\hline 130 y superior & Muy superior \\
\hline $120-129$ & Superior \\
\hline $110-119$ & Medio-alto \\
\hline $90-109$ & Medio \\
\hline $80-89$ & Medio-Bajo \\
\hline $70-79$ & Limite \\
\hline 69 e inferior & Muy bajo \\
\hline
\end{tabular}

Fuente: Bayley (2015).
Conforme a los resultados alcanzados, se manifiesta como la media obtenida en el área comunicativa-lingüística alcanza la misma puntuación en los tres análisis realizados ( $\overline{\mathbf{X}} 87.6)$ (rango: 47-127), independientemente del número de sujetos, situándose en un nivel medio-bajo (rango: 80-89). En el primer análisis se incluyó la cohorte total de 48 participantes, nacidos entre la semana 35 y la 41; en el segundo análisis, compuesto por 44 participantes, se excluyó a aquellos que nacieron prematuramente (35-36 semanas de EG) y tardíamente (41 semana de EG), mientras que el tercer análisis se realizó incluyendo únicamente a aquellos que fueron excluidos en el segundo análisis, es decir, aquellos que presentaron un nacimiento prematuro (35-36 semanas de EG) y tardío (41 semana de EG). Lo mismo sucede con las puntuaciones logradas en el área motora, aunque varían mínimamente entre el primer ( $\overline{\mathbf{X}} 88.7$ ) (rango: 46-127) y el segundo análisis (文89) (rango: 46-127), sin suceder lo mismo en el tercero ( $\overline{\mathbf{X}} 85.6)$ (rango: 67-100).

La comparativa de los resultados alcanzados en el neurodesarrollo de los menores se muestra en el gráfico 2 aunque, es preciso mencionar, que las áreas en las que se encuentran lentificaciones, como la motora y la del lenguaje, están compuestas de sub-áreas como la motricidad fina, la gruesa, el lenguaje comprensivo y el expresivo.

La Clasificación Internacional del Funcionamiento de la Discapacidad y de la Salud (CIF-IA) (OMS, 2011) registra que el proceso de adquisición del lenguaje debe ir acompañado del desarrollo de la capacidad para representar personas, objetos, eventos y sentimientos mediante palabras. Sin embargo, al valorar a los menores con estas anomalías crónicas se observa como obtienen un desarrollo inferior en el lenguaje expresivo, pues son capaces de señalar aquellos objetos mencionados por el examinador, pero incapaces de nombrarlos cuando se invierten las acciones, coincidiendo con los resultados obtenidos en la clasificación cualitativa de Bayley-III (2015).

El dominio de movilidad, según la CIF-IA (OMS, 2011), alude a la necesidad de adoptar cambios posturales básicos, tales como de estar sentado a estar de pie. En la motricidad gruesa de los participantes de este estudio, a una edad 
media de 23 meses, se identifica falta de coordinación en la marcha, pérdida de equilibrio en la carrera y en el paso de sedestación a bipedestación, entre otras cosas. Asimismo, la misma Clasificación, recoge la necesidad realizar acciones coordinadas a través de la manipulación de objetos, utilizando el uso fino de la mano. Sin embargo, los infantes evaluados muestran ausencia de la pinza, pues tratan de coger objetos únicamente utilizando la palma de la mano, lo que es un indicador de la dificultad presente en esta subárea.

Gráfico 2. Resultados de los análisis de las áreas del neurodesarrollo.

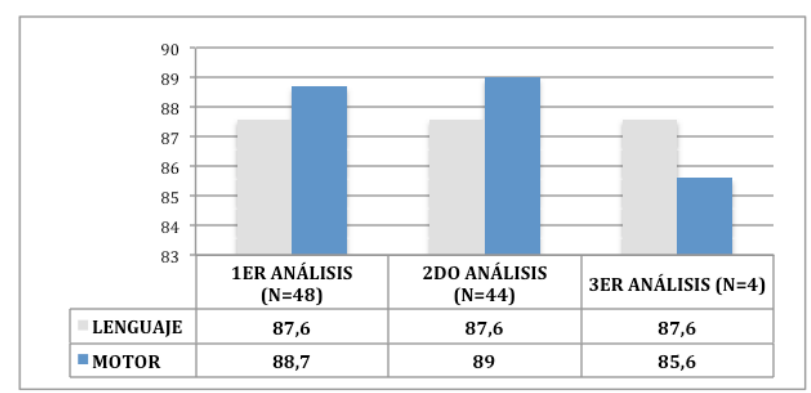

Fuente: elaboración propia.

Con base a los resultados alcanzados, y teniendo en cuenta el objetivo del estudio, los criterios biomédicos planteados (EG, PC, talla y peso), que adquieren incidencia en la población prematura, parecen no estar implicados con las lentificaciones encontradas en las áreas del desarrollo de los menores con patología cardiaca. Por este motivo, se incorporan nuevas variables a este análisis, pertenecientes a criterios biomédicos propios de la población con cardiopatías congénitas, en una submuestra de 12 participantes. Estos criterios hacen referencia al tiempo de cirugía por circulación extracorpórea (CEC), el cual se sitúa en promedio de $\overline{\mathbf{X}} \mathbf{1 5 2} \mathrm{min}$. (rango: 80-226); a la duración del clampaje ( $\overline{\mathbf{X}} 86$ min) (rango: 11-132); al tiempo de ingreso en UCIN ( $\overline{\mathbf{X}} 21$ días)(rango: 6-66), y al tiempo transcurrido desde la intervención quirúrgica hasta la valoración del neurodesarrollo ( $\overline{\mathbf{X}} 13.5$ meses) (rango: 9-17 meses). Esto sugiere que las lentificaciones encontradas en las áreas de desarrollo pueden estar asociadas a estas variables biomédicas. Sin embargo, es necesario ampliar la muestra del estudio para poder realizar correlaciones entre las variables biomédicas y las de desarrollo, a fin de conocer el grado de incidencia entre unas y otras.

\section{Conclusiones}

El principal objetivo de la Cardiología Infantil es garantizar la vida de los infantes con cardiopatías congénitas (Brotons, 2015). Cuando la anomalía congénita se corrige o se mitiga y el bebé prosigue con su desarrollo evolutivo, es cuando se empiezan a observar retrasos en los mismos (Cartón y Gutiérrez-Larraya, 2016). A priori, y en referencia a este estudio, las lentificaciones pueden encontrarse en el área motora y del lenguaje a edades tempranas, antes de los 42 meses de edad. Esto, según la literatura científica, está provocando dificultades de aprendizaje en áreas instrumentales como matemáticas que junto con los problemas encontrados en las funciones ejecutivas, en la lectoescritura, comprensión..., las cuales se hacen más evidentes en procesos superiores, repercuten en el desarrollo académico del menor con cardiopatía congénita (Matsuzaki et al., 2010; Mulkey et al., 2014).

Los menores de este estudio carecen de anomalías cromosómicas asociadas y/o de síndromes genéticos, pero en la recogida de la muestra actual se contó con participantes prematuros. Algunos autores indican que los menores con cardiopatías tienen un desarrollo similar a los prematuros (Esquivel-Hernández et al., 2013); sin embargo, las variables inductoras de comorbilidad en los prematuros no son extrapolables a la población con CC. Por tanto, los factores que pueden incidir en el posterior neurodesarrollo de los menores con CC en etapa infantil, están más relacionados a variables biomédicas peri o post quirúrgicas, tal y como parece mostrarse en la submuestra de 12 participantes, pero es necesario ampliar la cohorte del estudio para poder confirmar los resultados.

Teniendo en cuenta la importancia de este tema, la sensibilidad actual ante las poblaciones de alto riesgo de presentar una discapacidad, es necesario garantizar una calidad de vida óptima para estos menores. Por ende, es necesario profundizar en estudios con mayor tamaño muestral para garantizar un abordaje interdisciplinar de calidad, tanto desde el ámbito sanitario como desde el educativo. 


\section{Referencias}

Albert, D. (2019). Niakoro. Recuperado el 1 de marzo de 2019. https://www.niakoro.com/es/

Área del Corazón Infantil HGUGM. (2017). Corazón normal. Recuperado el 6 de marzo de 2019. https://www.cardiopedhgugm.com/cardiopat\%C3\%ADas-congénitas/corazón-normal/

Asociación de Anomalías y Malformaciones Dentofaciales (AAMADE). 2012. Síndrome de Edwars. Recuperado el 3 de junio de 2019. http://www.aamade.com/casos-clinicos/sindrome-deedwards.html

Bayley, N. (2015). Escala de Desarrollo Infantil Bayley-III. España: Pearson

Brotons, A. (2015). El desarrollo de la cardiología pediátrica española y su impacto en el manejo de las cardiopatías congénitas. Anales de Pediatría, 83(5): 295-296.

Cartón, A. y Gutiérrez-Larraya, F. (2016). Cardiopatías congénitas: evolución con la edad y seguimiento en Atención Primaria. Pediatr Integral, XX (8): 539-547.

Chen C, Harrison T, Heathcock J. (2015). Infants with complex congenital heart diseases show por shortterm memory in the mobile paradigm at 3 months of age. Infant Behav Dev, 40:12-19.

Children's Health. (2019). Comunicación interventricular (CIV). Recuperado el 6 de marzo de 2019. https://www.stanfordchildrens.org/es/topic/default?id=comunicacininterventricularciv-90-P04932

Chidren's Health. (2019). Comunicación Interauricular (CIA). Recuperado el 6 de marzo de 2019. https://www.stanfordchildrens.org/es/topic/default?id=comunicaci243n-interauricular-cia-90-P04869

Dittrich, H., Bührer, C., Grimmer, I., Dittrich, S., Abdul-Khaliq, H. \& Lange, P. (2003). Neurodevelopment at 1 year of age in infants with congenital heart disease. Heart, 89(4): 436 -441.

Esquivel-Hernández, F., Pliego-Rivero, F., Mendieta-Alcántara, G., Ricardo-Garcell, J. \& Otero-Ojeda, G. (2013). Alteraciones electroencefalográficas y del neurodesarrollo en niños portadores de cardiopatías congénitas severas. Estudio preliminar. Gac Med Mex, 149 (6): 605-612.

Fundación Menudos Corazones (MMCC). (2019). Menudos Corazones. Fundación de ayuda a los niños con problemas de corazón. Recuperado el 1 de marzo de 2019. https://www.menudoscorazones.org

Fundación Salud Infantil. (2019). Cardiopatías congénitas en el recién nacido. Recuperado el 6 de marzo de 2019.https://www.fundacionsaludinfantil.org/cardiopatias-congenitas-en-el-recien-nacido/

Gerstle M, Beebe D, Drotar D, Cassedy A, Marino B (2016). Executive Functioning and School Performance among Pediatric Survivors of Complex Congenital Heart Disease. J Pediatr, 173:154-9.

Goldsworthy, M., Franich-Ray, C., Kinney, S., Shekerdemian, L., Beca, J. \& Gunn, J. (2016). Relationship between Social-Emotional and Neurodevelopment of 2-Year-Old Children with Congenital Heart Disease. Congenit Heart Dis, 11(5): 378-385.

KidsHealth. (2019). Recuperado el 1 de marzo de 2019. https://kidshealth.org/es/parents/grownewborn-esp.html

Mayo Clinic. (2019). Síndrome DiGeorge. Recuperado el 1 de junio de 2019. https://www.mayoclinic.org/es-es/diseases-conditions/digeorge-syndrome/symptomscauses/syc-20353543

Marino, B., Lipkin, P., Newburguer, J., Peacock, G., Gerdes, M., Gaynor, J., Mussatto, K., Uzark, K., Goldberg, C., Johnson, W., Li, J., Smith, S., Bellinger, D. \& Mahle, W. (2012). Neurodevelopmental outcomes in children with congenital heart disease: evaluation and management: a scientific statement from the American Heart Association. Circulation, 126(9): 1143-72.

Martínez-Biarge, M. et al. (2013). Neurodevelopmental outcome in children with congenital heart disease. Seminars in Fetal \& Neonatal Medicine, 5 (18): 279-285.

Matsuzaki, T., Matsui, M., Ichida, F., Nakazawa, J., Hattori, A., Yoshikosi, K., Miyazaki, M., Fujii, M., Hagino, I., Kagisaki, K. \& Yagihara, T. (2010). Neurodevelopment in 1-year-old Japanese infants after congenital heart surgery. Pediatrics International, 52(3): 420- 427.

McGrath E, Wypij D, Rappaport L, Newburger J, Bellinger D. (2004). Prediction of IQ and achievement at age 8 years from neurodevelopmental status at age 1year in children with D-transposition of the great arteries. Pediatrics, 114(5):572-576. 
MedlinePlus. (2019). Biblioteca Nacional de Medician de los EE.UU. Recuperado el 1 de marzo de 2019. https://medlineplus.gov/spanish/ency/article/001104.htm

MedlinePlus. (2019). Transposición de las Grandes Arterias. Recuperado el 7 de marzo de 2019. https://medlineplus.gov/spanish/ency/article/001568.htm

Miatton M, De Wolf D, François K, Thiery E, Vingerhoets G. (2007). Neuropsychological performance in school-Aged children with surgically corrected congenital heart disease.J Pediatrics:73-78.

Mulkey, S., Swearingen, C., Melguizo, M., Reeves, R., Rowell, J., Gibson, N., Holland, G., Bhutta, A. \& Kaiser, J. (2014). Academic proficiency in children following early congenital heart disease surgery. Pediatr Cardiol, 35(2): 344-352.

Organización Mundial de la Salud. (2019). Anomalías congénitas. Recuperado el 28 de enero de 2019. https://www.who.int/es/news-room/fact-sheets/detail/congenital-anomalies

Organización Mundial de la Salud. (2011). Clasificación Internacional del Funcionamiento, de la Discapacidad y de la Salud: versión para la Infancia y la Adolescencia (CIF-IA). Recuperado el 17 de abril de 2020. https://apps.who.int/iris/handle/10665/81610

Perez-Lescure, J., Mosquera, M., Latasa, Pello y Crespo, D. (2018). Mortalidad de las cardiopatías congénitas en España durante 10 años (2003-2012). Anales de Pediatría, 88(5): 237-298.

Quero, M. y Sánchez, I. (2006). Capítulo II. Tipos de Cardiopatías . En Santos, J. (ED.), Manual para padres de niños con cardiopatía congénita (pp. 76-106). España: SECPCC

Rollins C, Newburger J. Neurodevelopmental outcomes in congenital heart disease. Cardiology Patient Page. 2014; 130: 124-126.

Salamanca-Zarzuela, B., Morales-Luego, F., Alcalde-Martín, C. y Centeno-Malfaz, F. (2018). Desarrollo psicomotor en pacientes con cardiopatía congénita grave. Rev. Neurología, 66: 409-414.

Sananes R, Manhliot C, Kelly E, et al. (2012). Neurodevelopmental outcomes after open heart operations before 3 months of age. Pediatric Cardiac, 93:1577-1583.

Sarrechia, I., Miatton, M., François, K., Gewillig, M., Mayns, B., Vingerhoets, G. \& de Wolf, D. (2015). Neurodevelopmental outcome after surgery for acyanotic congenital heart disease. Res Dev Disabil, 45-46: 58-68.

Solar, A. y García, L. (2010). Alimentación en el cardiópata. En SEGHNP-AEP. (Ed.), Protocolos diagnósticoterapéuticos de Gastroenterología, Hepatología y Nutrición Pediátrica (pp. 348-352). Madrid: Ergon.

Schaefer C, Von Rhein M, Knirsch W, et al. (2013). Neurodevelopmental outcome, psychological adjustment and quality of life in adolescents with congenital heart disease. DMCN, 55:1143-1149.

Williams, J. et al. (2015). Neurodevelopmental outcomes after cardiac surgery in infancy. Pediatrics, 135(5): 817-825. 